\title{
GESTIÓN DEL RIESGO DE DESASTRE EN EL CARIBE MEXICANO. EL CASO DE ESTUDIO DE CHETUMAL, QUINTANA ROO
}

Rosalía Chávez Alvarado ${ }^{1, *}$

\section{RESUMEN}

El presente trabajo tiene por objetivo explicar el papel que tienen las instituciones de gobierno municipal en la gestión del riesgo de desastre en ciudades costeras del caribe mexicano. La ciudad seleccionada fue Chetumal, por ser la capital del estado de Quintana Roo y concentrar los poderes institucionales, así como, ser la cuna de las decisiones gubernamentales. Se tomó un grupo de funcionarios públicos, como caso de estudio, seleccionados por su experiencia ante las contingencias por huracán. Los resultados se recopilaron con una entrevista semiestructurada, para conocer cómo se lleva a cabo la gestión del riesgo de desastres en una ciudad donde hay una amenaza constante de fenómenos hidrometeorológicos. Los resultados muestran la forma en que se entrelazan las instituciones de tres niveles de gobierno para la gestión del riesgo, la estructura de jerarquías, los niveles institucionales y sus atribuciones, así como el reconocimiento de algunas limitantes al trabajo ante los desastres que podrían ser reformadas para ir construyendo una región resiliente.

\section{PALABRAS CLAVE}

Gestión del riesgo, Resiliencia, Adaptación, Instituciones

\section{DISASTER RISK MANAGEMENT IN THE MEXICAN CARIBBEAN. THE CASE STUDY OF} CHETUMAL, QUINTANA ROO

\section{ABSTRACT}

The objective of this paper is to explain the role of municipal government institutions in disaster risk management in coastal cities of the Mexican Caribbean. The selected city was Chetumal, for being the capital of the state of Quintana Roo and concentrating the institutional powers, as well as, being the cradle of the governmental decisions. A group of public officials was taken as a case study, selected for their experience in hurricane contingencies. The results were compiled with a semi-structured interview, to find out how disaster risk management is carried out in a city where there is a constant threat of hydrometeorological phenomena. The results show the way in which the institutions of three levels of government are intertwined for risk management, the structure of hierarchies, the institutional levels and their attributions, as well as the recognition of some limitations to work in the face of disasters that could be reformed to build a resilient region.

\section{KEYWORDS}

Risk management, Resilience, Adaptation, Institutions

1. División de Ciencias e Ingeniería, Universidad de Quintana Roo, Chetumal, México.

*Autor de correspondencia: rosaliadf@gmail.com

\section{RECIBIDO}

23 de mayo de 2018

\section{ACEPTADO}

22 de junio de 2018

\section{PUBLICADO}

25 de julio de 2018

\section{Formato cita}

Recomendada (APA): Chávez Alvarado, R. (2018). Gestión del riesgo de desastre en el caribe mexicano. El caso de estudio de Chetumal, Quintana Roo. Revista de Estudios Latinoamericanos sobre Reducción del Riesgo de Desastres REDER, 2(2), pp.46-6o

\section{(ब) $(\AA \otimes$}

Todos los artículos publicados en REDER siguen una política de Acceso Abierto y se respaldan en una Licencia CreativeCommons Atribución-NoComercial 4.0 Internacional.

\section{Revista de Estudios}

Latinoamericanos sobre Reducción del Riesgo de Desastres (REDER)

Diseño: Lupe Bezzina Tipografía: Hospital 


\section{INTRODUCCIÓN}

El entorno natural se modifica continuamente por acciones humanas, sin embargo, el cambio climático contribuye a establecer nuevas políticas públicas sobre la necesidad de gestionar el riesgo de desastre (prevención, atención, recuperación, reconstrucción y mitigación), debido a los eventos extremos que ocasiona (Morrison et al., 2017).

El incremento del riesgo de desastre por huracanes, es de especial relevancia en las ciudades costeras por dos causas principales, los informes del Panel Intergubernamental sobre Cambio Climático (IPCC, por sus siglas en inglés) en 2013, las señaló como aquellas de mayor afectación por fenómenos hidrometeorológicos, además, serán las ciudades con tasas importantes de crecimiento demográfico, así que, se torna evidente la urgencia de incrementar los estudios sobre las acciones institucionales de las poblaciones que habitan en esos espacios urbanos ( $\varnothing$ stergaard Nielsen \& Reenberg, 2010).

En México, al hablar de la gestión del riesgo de desastre, se mencionan conceptos, como la vulnerabilidad social y la resiliencia, ya que, a pesar de ser nociones que analizan procesos sociales y ambientales distintos, son resultado de un conjunto de acciones institucionales que prolongan los problemas estructurales que impiden obtener prominentes efectos al momento de diseñar, implementar y evaluar las políticas públicas en materia de gestión del riesgo de desastres.

El conocimiento de la gestión del riesgo de desastre por parte de los funcionarios públicos permitiría entender las necesidades reales ante las amenazas por fenómenos hidrometeorológicos. Un ejemplo simbólico es que se confunde la vulnerabilidad social con la pobreza, incluso se utilizan como sinónimos, se dejan de lado los aspectos estructurales que pueden ser más determinantes en la fragilidad humana, como el género, etnia, religión, política, medio ambiente, cultura, fenómenos hidrometeorológicos o menor respuesta institucional (Kelly \& Adger, 2000; Cutter, 2016; Chan et al., 2004; Jonkman et al., 2009).

Diversos estudios analizan factores sociales, económicos, ambientales y políticos sobre la capacidad de respuesta de las instituciones y su adaptación a la sucesión continua de desastres (Cutter et al., 2003; Chan et al., 2004; Brouwer et al., 2007; Balcu, 2010; Gupta et al., 2011), incluso hay trabajos sobre cómo medir la capacidad de respuesta mediante la infraestructura crítica, aumentando la posibilidad de implementar políticas públicas de acuerdo a los contextos ambientales de cada ciudad (Fekete et al., 2012; Fekete et al., 2015).

En países como México, la gestión del riesgo de desastres es un conjunto de políticas implementadas para atender, evacuar y recuperar a las comunidades afectadas por un desastre. En los funcionarios públicos recae la toma de decisiones, aplicación de presupuestos, por lo que este trabajo plantea, el supuesto de que, sus actuaciones y discurso institucional son la representación simbólica de un bagaje sobre la capacidad de adaptación que se transforma lenta y repentinamente, debido al cambio climático, plasmando la construcción limitada y parsimoniosa de una mínima capacidad de respuesta. El objetivo de este trabajo es enfocar el diagnóstico de la gestión del riesgo de desastres en la ciudad de Chetumal, analizando las respuestas sobre una entrevista semiestructurada cuyo enfoque fue indagar sobre la estructura institucional vertical y horizontal para la gestión del riesgo de desastre, sus avances y limitaciones. El trabajo se centra en la ciudad de Chetumal, parte de la región caribeña mexicana, que concentra los poderes estatales de Quintana Roo, México, donde definen las políticas de actuación durante la temporada de huracanes, aplicadas al resto de sus ciudades caribeñas costeras de turismo internacional, Tulum, Playa del Carmen, Cancún, Cozumel e Isla Mujeres.

\section{GESTIÓN DEL RIESGO Y RESILIENCIA URBANA}

Chan et al. (2014) afirman que la gestión del riesgo de desastre debiera producir un desarrollo sostenible, identificar la dinámica del sistema, su capacidad de responder y adaptarse al cambio climático, regresándolo a la estabilidad, a través de su auto-organización y experiencia, reflejando un sistema de regulación continua, la oportunidad de utilizar la experiencia para mejorar la capacidad de respuesta contribuye al concepto de resiliencia urbana.

La gestión del riesgo significa una serie de acciones que deberían evitar llegar a la catástrofe, cuando ya se está en ella se define, como gestión del riesgo de desastre, sin embargo, el contexto social, ambiental y político, influye en los resultados. Existe la prevención, atención, recuperación y reconstrucción, como fases de la gestión del riesgo de desastres, en cada una, la mayoría de los 
gobiernos nacionales y locales han implementado un esquema de actuación a fin de dar respuesta y apoyar a la comunidad. Las instituciones internacionales proponen la transversalización de la gestión del riesgo de desastre a fin de integrarla en todos los aspectos sociales, ambientales y políticos de un país (PNUD, 2014). Aún es esencial considerar a la población en general, como parte de una estrategia social de gestión del riesgo.

Otros estudios, enfatizan la necesidad de anexar una gobernanza climática global que signifique el trabajo de gobiernos de todo el mundo y, de los actores locales que apoyan en la conciencia generalizada para acciones sociales (Demares, 2016). La comunicación entre la sociedad civil y las instituciones encargadas de la gestión del riesgo de desastre es elemental para lograr avanzar en la construcción de la resiliencia.

La forma de actuación institucional es distinta por la representación en que culturalmente se comprende el impacto extremo y sus consecuencias, de ello depende la priorización de procesos, procedimientos y la forma operacional de las organizaciones. Seleccionar los factores estructurales más importantes para la sociedad en general y asignar los presupuestos. Es un proceso multifactorial. Las discusiones actuales proponen tomar como referencia medir la resiliencia a fin de evaluar o calcular la efectividad de las políticas públicas, los protocolos de actuación y las intervenciones diseñadas a fin de gestionar el riesgo de desastre, de manera, que rápidamente se logre la recuperación y se inicie la reconstrucción. Para ello, es primordial analizar la situación actual a fin de contar con un punto de referencia (Elliot et al., 2010; Cutter et al., 2010), con esto nos referimos a la construcción de bases de datos para contar con cifras medibles o elementos cualitativos de comparación a futuro.

Existen escasos, pero sugestivos estudios documentados sobre la comprensión y perspectiva de los empleados gubernamentales y civiles organizados, acerca de la gestión del riesgo de desastre (Cutter et al., 2010; Chan et al., 2014). La concepción institucional de la gestión del riesgo de desastre, su cultura y perspectiva de grupos vulnerables define la forma de actuar y responder ante un riesgo. En ello, influyen la transmisión de un mensaje, la forma en que se coordina con sus compañeros de trabajo, la claridad de objetivos y medios de apoyo con que se cuenta, priorizar acciones justificadas socialmente, considerar que los funcionarios también requerirán resolver emergencias familiares, flexibilizar la tensión de los protagonistas en el apoyo a sus propias familias genera liderazgo por la confianza y compromiso por aligerar la incertidumbre doméstica. Además, refleja el nivel de conciencia que tienen los líderes de las instituciones gubernamentales sobre las necesidades de la comunidad completa. (Nilakant et al., 2014)

La utilización de indicadores socioeconómicos, como la población y viviendas afectadas, número de personas que utilizan los refugios, capacidad de respuesta de la población por barrios, podría simplificar y comunicar la realidad en tiempo y espacio definido (Freudenberg, 2003), observar las capacidades de respuesta y de adaptación a los cambios climáticos en el tiempo, sería una de las herramientas políticas sobre la priorización de acciones, campos, grupos, lugares, etc. (Cutter et al., 2010). La gobernanza comienza a ser otro atractivo para los estudios sobre gestión del riesgo, ya que es evidente que la participación social, la representación, equidad, legitimidad, responsabilidad, innovación y eficiencia estén presentes. La gobernanza policéntrica, tal como la describen Morrison y sus colegas (2017) expande el concepto para integrar gobiernos afectados por un evento del cambio climático, para tomar decisiones, integrar presupuestos y recursos humanos, a fin de operar sin una autoridad central o federal predominante, pero con orden; así, como compartir experiencias exitosas. Se han encontrado ejemplos diversos de corrupción, jerarquización en la atención a fin de segregar grupos o ciudades, empoderamiento negativo, aprovechamiento de recursos locales limitando el resultado de la gestión del riesgo efectiva (Nilakant et al., 2014, Morrison et al., 2017), aumentando la desconfianza en las instituciones de gobierno.

El liderazgo es una característica escasamente estudiada en la gestión del riesgo de desastre, el comportamiento de los miembros de un grupo, en este caso, de los empleados gubernamentales sería preponderante para que la comunidad participe y se logre un proceso de aprendizaje personal que vaya construyendo una cultura de apoyo, compromiso, colaboración y mitigación, finalmente, la construcción de una sociedad resiliente, "mejor respuesta, recuperación más rápida y renovación positiva" (Nilakant et al., 2014). En este proceso se incluye la forma de ejercer el régimen de responsabilidad administrativa frente al daño ambiental, evitando la permisividad que lesiona el 
entorno y, muchas veces conduce a incrementar riesgos por algún fenómeno natural, como los huracanes. Para Leyva (2016) es complicado el análisis de la responsabilidad (civil, administrativo, penal o ambiental), porque se interpone el demostrar la existencia de una lesión, cómo definir el daño ecológico, siendo éste concebido como un daño abstracto, porque no recae en derechos personales, animales, de género, entre otros. Es más reconocido como un daño colectivo o algo subjetivo.

El daño ambiental presenta una perspectiva dinámica y holística, cuántas personas son afectadas y cuántas afectan, la duración de la afectación, la magnitud del impacto, estamos ante una diferenciación de realidad práctica, donde la construcción de la ciudad es responsabilidad social, empresarial e institucional, con finalidades jurídicas distintas, con causas y consecuencias disímiles para cada caso. Sin embargo, también existe la responsabilidad subjetiva, ya que la ley permite la duda sobre la negligencia o no, el resarcir todos o algunos de los daños, ya que la actividad puede llevar daños morales, patrimoniales, directos e indirectos (Leyva, 2016; Demares, 2016). Por lo anterior, la responsabilidad de las instituciones gubernamentales administrativas se debe apegar a:

“a) El primero de los elementos es la lesión patrimonial equivalente a daño o perjuicio en la doble modalidad de lucro cesante o daño emergente. b) En segundo lugar, la lesión se define como daño ilegítimo. c) El vínculo entre la lesión y el agente que la produce, es decir, entre el acto dañoso y la Administración, implica una actuación del poder público en uso de potestades públicas. d) Finalmente, la lesión ha de ser real y efectiva, nunca potencial o futura, pues el perjuicio tiene naturaleza exclusiva con posibilidad de ser cifrado en dinero y compensado de manera individualizable, debiéndose dar el necesario nexo causal entre la acción producida y el resultado dañoso ocasionado".

(Leyva, 2016, p.119)

Por lo que resulta complicada la acusación a una autoridad o persona que haya obtenido un permiso para construcción o cambio de usos de suelo. Empero, es posible acreditar daños colaterales generados por alguna acción pre-establecida en la ley nacional, sin embargo, hasta ahora han sido escasos los ejemplos al respecto. La construcción de un marco legal ambiental es parte de un proceso constructivo en la gestión del riesgo, siendo la etapa de recuperación y reconstrucción donde la adaptación es más evidente (Nilakant et al., 2014, Chun et al., 2014).

La adaptación debe entenderse como el periodo de ajuste de las actividades humanas o de los ecosistemas a condiciones climáticas dinámicas (IPCC, 2007). Actualmente, la Comisión Económica para América Latina y el Caribe (CEPAL), Banco Interamericano de Desarrollo (BID) y World Wildlife Fund (WWF) mencionan la Convención Marco de las Naciones Unidas sobre el Cambio Climático (CMCC), el Mecanismo de Desarrollo Limpio (MDL) y más recientemente el Fondo de Adaptación (FA) como acciones de respuesta de adaptación al cambio climático en América Latina y el Caribe para disminuir los efectos sobre zonas costeras, recursos hídricos, biodiversidad y salud. Estos programas financian acciones tendientes a prevenir y mitigar los efectos climáticos en distintos lugares del continente americano (Vergara et al., 2014).

Se ha identificado a la adaptación como una reacción anticipada a estímulos externos, tales como el estrés ambiental, la perspectiva de este trabajo se enfoca en una adaptación al cambio ambiental centrándose en un marco útil para identificar la gestión del riesgo de desastre, como respuesta y las políticas aplicadas, identificar los ajustes y acciones transformativas, esto a través de escalas, la ciudad, el municipio y el estado. Sin perder de vista la participación social activa, las redes civiles y, cómo el gobierno les transmite su experiencia institucional (Elliot et al., 2010). Por último, estudios muestran que el uso de las Tecnologías de la Información y Comunicación (TIC) aumenta el intercambio de conocimientos mediante la utilización de los teléfonos móviles y las redes sociales, construyendo una base de datos participativa en tiempo real, con las personas como agentes activos y sin la dependencia de las estructuras formales de enlace. Es patente que la información en las redes sociales podría tener un potencial para complementar la situación tradicional, informar y comparar los comentarios hasta que se forme una base de datos significativa. Existen ejemplos de la efectividad de las redes sociales, como Facebook y twitter ante desastres, como Haití en 2010, Japón en 2011 y el huracán Sandy en 2012 (Shibuya, 2017). 


\section{CASO DE ESTUDIO}

La ciudad de Chetumal se localiza al sur del estado de Quintana Roo, en el municipio de Othón P. Blanco, en el país de México, colindando con Belice (ver Figura 1). En este estado se ubican otros puntos turísticos de índole internacional que también se ven afectados por los huracanes, sin embargo, se seleccionó a la ciudad de Chetumal ya que está en el mismo nivel de amenaza y es el centro de ubicación de las instituciones estatales. Chetumal presenta una característica relevante para el análisis sobre gestión del riesgo de desastre, concentra los poderes políticos que son los que decidirán acciones en el momento de verse en situación de peligro. La forma en que las autoridades locales decidan enfrentar el riesgo, será un ejercicio del que dependen otras ciudades del estado, por ser la capital y concentrar los poderes de decisión.

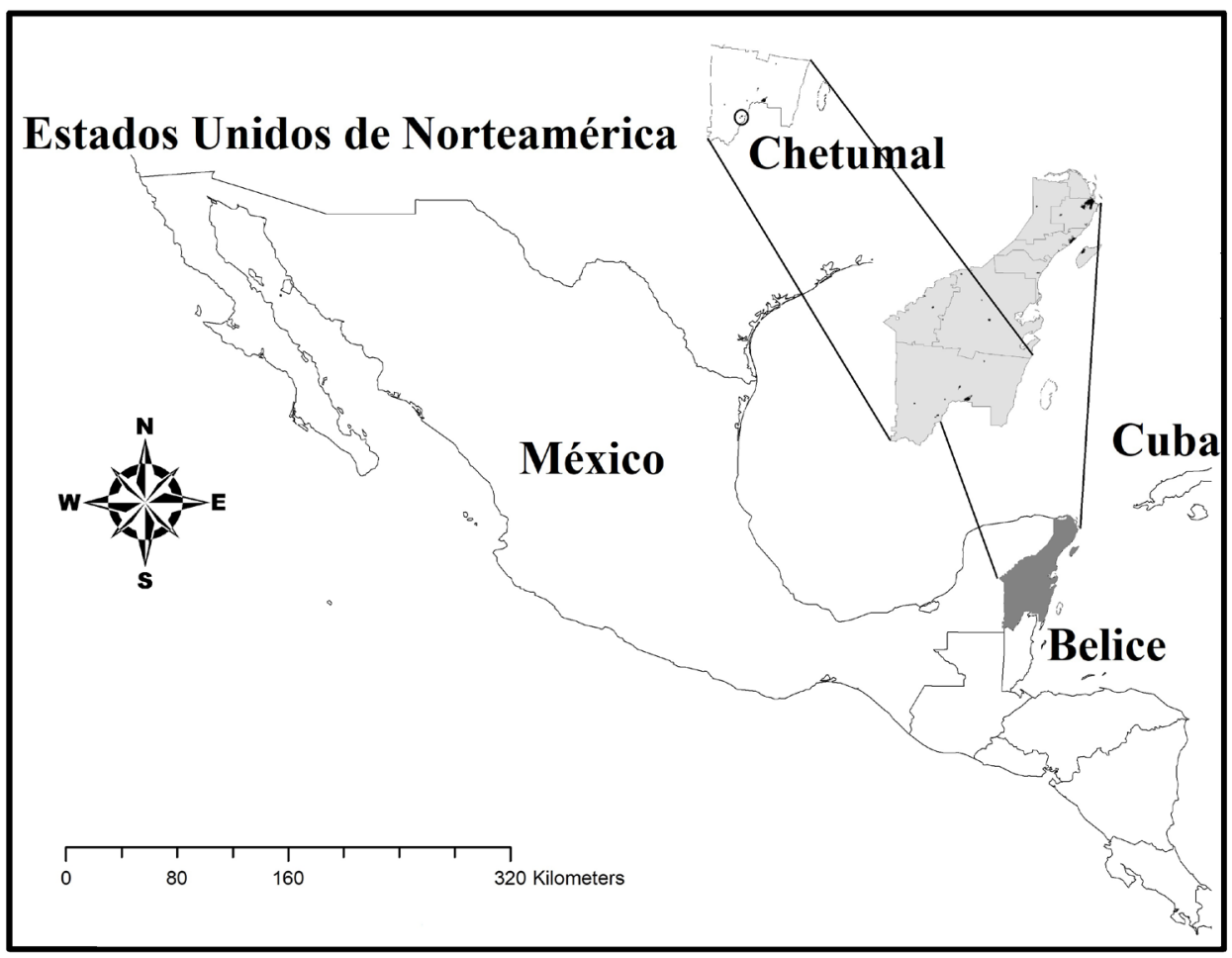

Figura 1. Ubicación geográfica de Chetumal en el contexto nacional y estatal Fuente: Autora, 2018, basada en INEGI (2016)

Las ciudades del caribe mexicano (Cancún, Cozumel, Tulum, Playa del Carmen y Chetumal) presentan tasas de crecimiento demográfico, en promedio ( $12.5 \%$ ) y por encima de la media nacional (1.4\%) (INEGI, 2015), las personas de estas ciudades reconocen que los problemas prioritarios son, la oportunidad de empleo, la incidencia de huracanes, además, los bajos salarios, la escasez de vivienda y oportunidades de obtenerla, los servicios con los que cuentan, el agua no es potable en ninguna ciudad (INE, 2000), la corrupción y la inseguridad (González \& Torruco, 2015). Aunado a ello, en los planes de desarrollo urbano se reconoce la problemática de la recolección y tratamiento de los desechos sólidos urbanos. Otra situación imperante, es la visión de ocupación territorial y su coordinación con políticas sectoriales, cuyo enfoque difiere de disminuir la tasa de pobreza y desempleo (Riffo, 2013).

El cambio climático se plasma territorialmente en Chetumal por sus impactos en el entorno natural, se alteran los ecosistemas más frágiles y disminuye ofrecer beneficios sociales, económicos y ambientales. Las modificaciones fisiográficas, el aumento en el nivel del mar, la presencia de sargazo, la erosión de la bahía y playas aledañas, las construcciones artificiales sufren desequilibrios y aumentan las áreas ambientales vulnerables (González \& Torruco, 2015). Esto conduce a suponer que, si la construcción del riesgo es social, así mismo se construye la gestión del riesgo de desastre y la capacidad de adaptación. La oportunidad de realizar diagnósticos sobre estos temas puede contribuir, como una estrategia en la producción de políticas públicas mejorando la capacidad de respuesta. 


\section{METODOLOGÍA}

Es un trabajo exploratorio, de tipo inductivo cualitativo para recolectar y analizar datos sobre las formas de desempeño de funcionarios públicos del nivel municipal y estatal. El desempeño de los empleados gubernamentales de mandos medios es fundamental (directores de área, coordinadores y supervisores), son empleados que acumulan años y experiencia en los puestos, y su conocimiento de eventos anteriores y las estrategias de respuesta permite identificar si la gestión del riesgo del desastre institucional avanzó en la capacidad de respuesta y adaptación (Cutter et al., 2010; Chan et al., 2014; Nilakant et al., 2014). La forma en que se seleccionó a los funcionarios públicos fue a través de la técnica "bola de nieve", que usa un muestreo no probabilístico manejada por los investigadores para identificar a los sujetos potenciales en estudios en donde los sujetos son difíciles de encontrar identificando a quienes sobresalieron en alguna experiencia sobre gestión del riesgo de desastre ante huracanes. El objetivo fue entrevistarlos con un guión semi-estructurado (entrevistas a profundidad) que permitió obtener información sobre el desempeño institucional, conocimiento de conceptos, relación de instituciones con la sociedad civil, avances y limitaciones en la gestión del riesgo de desastre en la ciudad de Chetumal. Las entrevistas fueron aplicadas en mayo del 2017 a marzo de 2018, en las oficinas de los funcionarios. El total de personas entrevistadas fue de diez funcionarios, ya que se logró el punto de saturación. Las entrevistas se grabaron en audio para su transcripción y, fueron analizadas mediante el uso de Atlas.ti, a fin de contextualizar algunos conceptos sobre la gestión del riesgo de desastre, evaluando los códigos y codificación de validez y confiabilidad en el proceso. Posteriormente, se organizó por temas emergentes destacados hacia la capacitación sobre la gestión del riesgo de desastre, la falta de liderazgo con la sociedad, la permisividad que afecta el desempeño de otras instituciones de gobierno, los distintos enfoques en la gestión del riesgo de desastre entre niveles de gobierno y la producción de información que permita tomar decisiones.

\section{LA GESTIÓN DEL RIESGO DE DESASTRE EN CHETUMAL, QUINTANA ROO.}

La primera fase del cuestionario exponía el conocimiento de conceptos, riesgo, vulnerabilidad y resiliencia. Comúnmente riesgo fue reconocido, como "un suceso que expone a la población a un nivel de fragilidad". La vulnerabilidad fue definida, como "la capacidad de las personas frágiles a enfrentar un peligro", mientras que la resiliencia fue determinada, como "la capacidad de enfrentar y recuperarse rápidamente de un peligro", todos los entrevistados lo enmarcaron como un tema ambiental, reconocieron que existe el cambio climático, sin embargo, existe escasa información de afectaciones y qué hacer a nivel local.

A pesar de que las respuestas mostraron una sencillez de los conceptos, al ir adentrando en la conversación, fue evidente la falta de algunas consideraciones de los entrevistados, por ejemplo, la vulnerabilidad social se relaciona con las transformaciones sociales, políticas e institucionales que impactan en el bienestar de la población (Busso, 2012), así que, además de las personas frágiles, la población completa puede ser vulnerable, principalmente, si sus instituciones y las políticas públicas deterioran las herramientas internas y externas para hacer frente a cualquier riesgo.

La forma en que han definido los conceptos revela que hace falta mayor difusión de información, de trabajos e investigaciones que puntualicen lo que significa cada concepto, adentrarse en las discusiones desde distintas perspectivas, en qué consiste, qué implica para la gestión del riesgo, a fin de ampliar las acciones institucionales y subsanar las limitaciones que ellos mismos mencionan y se describen más adelante. Principalmente, cuando se alude a la asistencia a cursos de capacitación que, están lejos de ser socializados a todos los funcionarios públicos, limitando la comprensión de los nuevos paradigmas en la administración de una ciudad, tal como Nilakant y sus colegas (2014) mencionan, la forma en que se transmite la información es relevante para el éxito en la disminución de daños frente a los desastres.

En el contexto de la entrevista, algunos funcionarios públicos dejaron conocer que se han creado foros internacionales y nacionales sobre el tema de ordenamiento territorial, declaración de áreas protegidas, protección civil, así como, los mismos congresos que las universidades locales realizan. Sin embargo, los entrevistados aceptan que no siempre pueden asistir, que algunos son foros reducidos a cierto público, cuando las universidades hacen las invitaciones su asistencia es mínima, solo asisten como ponentes y, sin son invitados están solo en la inauguración y después se tienen que retirar por su agenda de trabajo.

También reconocen la falta de interés del público en general, la asistencia de la sociedad es 
mínima, hipotéticamente se puede deber a la forma en que se publicita el evento o el enfoque temático que no despierta el interés social.

"Se abrió un Foro Nacional de Protección Civil para el público en general, el tema principal era conocer cómo se generan los riesgos de incendio y qué hacer en caso de decidir ser voluntario. La mayoría de los asistentes fueron funcionarios, no asistieron a todos los cursos y pláticas, hubo poca asistencia de la gente, de personas o alumnos de escuelas, si bien, a veces los horarios no coinciden, creo que para la población en general no hay un interés de saber, de ayudar, de conocer, tampoco la chamba es toda nuestra".

(Protección Civil Estatal, Entrevista, 2018)

Quintana Roo muestra, como otros estados de México, un interés por desarrollar una gestión del riesgo de desastre ante huracanes, la continua apertura de foros, congresos, datos en los medios de comunicación, es una muestra del trabajo que se realiza sobre la comunicación del gobierno y sus instituciones hacia la ciudadanía. Lo que se debe de reconocer, es que desde 1955 los huracanes sólo se acercan a Chetumal, sin impactar en tierra, lo que hace confiar a la población (ver Tabla 1). Estos datos orientan a suponer dos situaciones: a) el gobierno estatal y local presentan un mínimo liderazgo que convoca a la población a ser partícipes de generar una efectiva gestión del riesgo, prevenir, atender, recuperarse y reconstruir $y$; b) por otro lado, parece que la convocatoria hacia la población logra nimios resultados, lo que hace suponer que la confianza de la población hacia la lejanía de enfrentar un huracán, podría ser un factor relevante en la construcción de su capacidad de respuesta.

\begin{tabular}{|c|c|c|c|}
\hline Nombre & Año & Categoría & Municipio \\
\hline JANET & 1955 & $\mathrm{H} 5$ & O.P. Blanco \\
\hline GILBERT & 1988 & H5 & Cozumel, Solidaridad \\
\hline DEAN & 2007 & $\mathrm{H} 5$ & Mahahual, O. P. Blanco \\
\hline CHARLIE & 1951 & $\mathrm{H} 4$ & Cozumel, Solidaridad \\
\hline CARMEN & 1974 & $\mathrm{H} 4$ & O.P. Blanco \\
\hline EMILY & 2005 & $\mathrm{H} 4$ & Cozumel, Solidaridad \\
\hline WILMA & 2005 & $\mathrm{H} 4$ & Cozumel, Solidaridad, B. Juárez \\
\hline SIN NOMBRE & 1903 & $\mathrm{H} 3$ & Cozumel, Solidaridad, B. Juárez \\
\hline SIN NOMBRE & 1909 & $\mathrm{H} 3$ & B. Juárez, Isla Mujeres \\
\hline ROXANNE & 1995 & $\mathrm{H} 3$ & F.C. Puerto, Solidaridad \\
\hline SIN NOMBRE & 1857 & $\mathrm{H} 2$ & B. Juárez, Isla Mujeres \\
\hline SIN NOMBRE & 1880 & $\mathrm{H} 2$ & Cozumel, Solidaridad, B. Juárez \\
\hline SIN NOMBRE & 1887 & $\mathrm{H} 2$ & Cozumel, Solidaridad, B. Juárez \\
\hline SIN NOMBRE & 1887 & $\mathrm{H} 2$ & B. Juárez, Isla Mujeres \\
\hline SIN NOMBRE & 1889 & $\mathrm{H} 2$ & F.C. Puerto \\
\hline SIN NOMBRE & 1893 & $\mathrm{H} 2$ & Cozumel, Solidaridad, B. Juárez \\
\hline SIN NOMBRE & 1895 & $\mathrm{H} 2$ & B. Juárez, Isla Mujeres \\
\hline SIN NOMBRE & 1909 & $\mathrm{H} 2$ & B. Juárez, Isla Mujeres \\
\hline SIN NOMBRE & 1916 & $\mathrm{H} 2$ & O.P. Blanco \\
\hline SIN NOMBRE & 1922 & $\mathrm{H} 2$ & Cozumel, Solidaridad \\
\hline SIN NOMBRE & 1933 & $\mathrm{H} 2$ & Cozumel, Solidaridad \\
\hline SIN NOMBRE & 1938 & $\mathrm{H} 2$ & B. Juárez, Isla Mujeres \\
\hline SIN NOMBRE & 1938 & $\mathrm{H} 2$ & Cozumel, Solidaridad \\
\hline SIN NOMBRE & 1942 & $\mathrm{H} 2$ & Cozumel, Solidaridad, B. Juárez \\
\hline HILDA & 1955 & $\mathrm{H} 2$ & F.C. Puerto \\
\hline BEULAH & 1967 & $\mathrm{H} 2$ & Cozumel, Solidaridad \\
\hline
\end{tabular}

(Continúa en la siguiente página) 


\begin{tabular}{llll} 
(Continuación) & Año & Categoría & Municipio \\
\hline SIN NOMBre & 1866 & H1 & O.P. Blanco \\
\hline SIN NOMBRE & 1870 & H1 & Costa desde el sur al norte \\
\hline SIN NOMBRE & 1873 & H1 & Isla Mujeres \\
\hline SIN NOMBRE & 1877 & H1 & Isla Mujeres \\
\hline SIN NOMBRE & 1879 & H1 & F.C. Puerto \\
\hline SIN NOMBRE & 1887 & H1 & Cozumel, Solidaridad, B. Juárez \\
\hline SIN NOMBRE & 1887 & H1 & B. Juárez, Isla Mujeres \\
\hline SIN NOMBRE & 1888 & H1 & B. Juárez, Isla Mujeres \\
\hline SIN NOMBRE & 1893 & H1 & Cozumel, Solidaridad, B. Juárez \\
\hline SIN NOMBRE & 1933 & H1 & O.P. Blanco \\
\hline SIN NOMBRE & 1934 & H1 & O.P. Blanco, F.C. Puerto \\
\hline SIN NOMBRE & 1944 & H1 & Cozumel, Solidaridad \\
\hline SIN NOMBRE & 1944 & H1 & B. Juárez, Isla Mujeres \\
\hline DOLLY & 1966 & H1 & O.P. Blanco, F.C. Puerto \\
\hline
\end{tabular}

Tabla 1. Huracanes ordenados por su categoría e impacto en Quintana Roo Fuente: Ihl \& Frausto (2014)

En otras instancias, como la Secretaría de Ecología del municipio de Chetumal (SE) y la Secretaría Estatal de Desarrollo Urbano y Vivienda de Quintana Roo (SEDUVI), se mencionó la presencia de asentamientos humanos informales que han absorbido una cantidad de inversión para evitar que las lluvias constantes provoquen algún desastre y, reconocen que hay permisividad de otras instancias gubernamentales para aceptar la presencia de estos asentamientos. Los entrevistados mencionaron que, a pesar de no contar con cifras, estiman que se ha invertido más en poner remiendos en la ciudad que en solucionar el problema de raíz, en generar una prevención y mitigación.

El proceso de adaptación en el estado de Quintana Roo, desde su ciudad capital Chetumal, muestra que las respuestas a las amenazas se limitan a cubrir el problema por un momento, operaciones donde están ausentes políticas públicas que favorezcan la acciones transformativas sobre la permisividad, legal e ilegal, donde los marcos normativos sean útiles en la identificación y mitigación de posibles amenazas y, sobre todo en una ausencia de construcción de redes civiles, foros de participación social, de recopilación de experiencias exitosas a nivel local o barrio, a fin de que la experiencia en cualquier ciudad de Quintana Roo, sirva para replicar como un modelo inicial de transformación futura que se adecue al contexto de cada ciudad.

Tal como lo mencionaron algunos autores, la adaptación, como parte de la resiliencia incluye un ajuste de las actividades humanas o ecosistemas a condiciones climáticas restauradas, a reaccionar anticipadamente a estímulos por el cambio climático, generar políticas públicas y a disminuir los impactos futuros, al menos evitar que sean tan drásticos a diferencia de los últimos desastres (IPCC, 2007; Elliot et al., 2010; Walter et al., 2014).

Existe un reconocimiento sobre los periodos de mayor posibilidad de amenaza de huracanes por el mar caribe, de junio a noviembre, empero, los entrevistados consideraron una adaptación ambiental al cambio climático, modificando la temporalidad, la frecuencia y la intensidad, ahora es menor predecible la temporada de lluvias, por lo que actividades como la agricultura de temporal se han reducido drásticamente. También reconocen que la población interviene negativamente en las acciones preventivas sobre la tala de árboles y limpieza en la vegetación, a fin de evitar que cuando llegue el huracán se convierta en un peligro.

"Por ejemplo, ahorita que ya se instaló el Comité, ahorita ya se está iniciando con la limpieza de los pozos de absorción, la recoja de basura, pasando información, diciéndole a la gente, de que por favor si tienen algún árbol en riesgo, repórtenlo. Entonces, mucha gente dice: ¡Ay no! A mi arbolito no le va a pasar nada. Pero cuando ya dicen el huracán lo tienes a tal hora, ya quieren incluso que vayan y te corten el árbol y obviamente, no se puede hacer así, aunque uno quiera, porque se tiene trabajo agendado".

(Secretaría de Desarrollo Urbano Municipal de Chetumal, Entrevista, 2017) 
Existe un reconocimiento, por parte de los entrevistados, sobre las omisiones en las revisiones periódicas, sanciones y acciones que responsabilicen a funcionarios y empresarios, además, de mencionar que hace falta realizar una propuesta integral sobre la planificación territorial. En este caso se comprueba que la vulnerabilidad social también se va construyendo, con un factor relevante que es el trabajo de las instituciones (Busso, 2012; Nilakant et al., 2014)

Protección Civil Estatal resulta ser la institución que establece la estrategia y atiende todas las emergencias, por lo tanto, reconoce que, a pesar de que tienen escaso personal, existe mínima rotación del mismo, lo que incrementa la experiencia, pero las situaciones de estrés los rebasan. Se observa un nivel jerárquico intermedio, entre el federal y el local, sin embargo, es solo normativo, escasamente llegan a ser operativos (ver Figura 2).

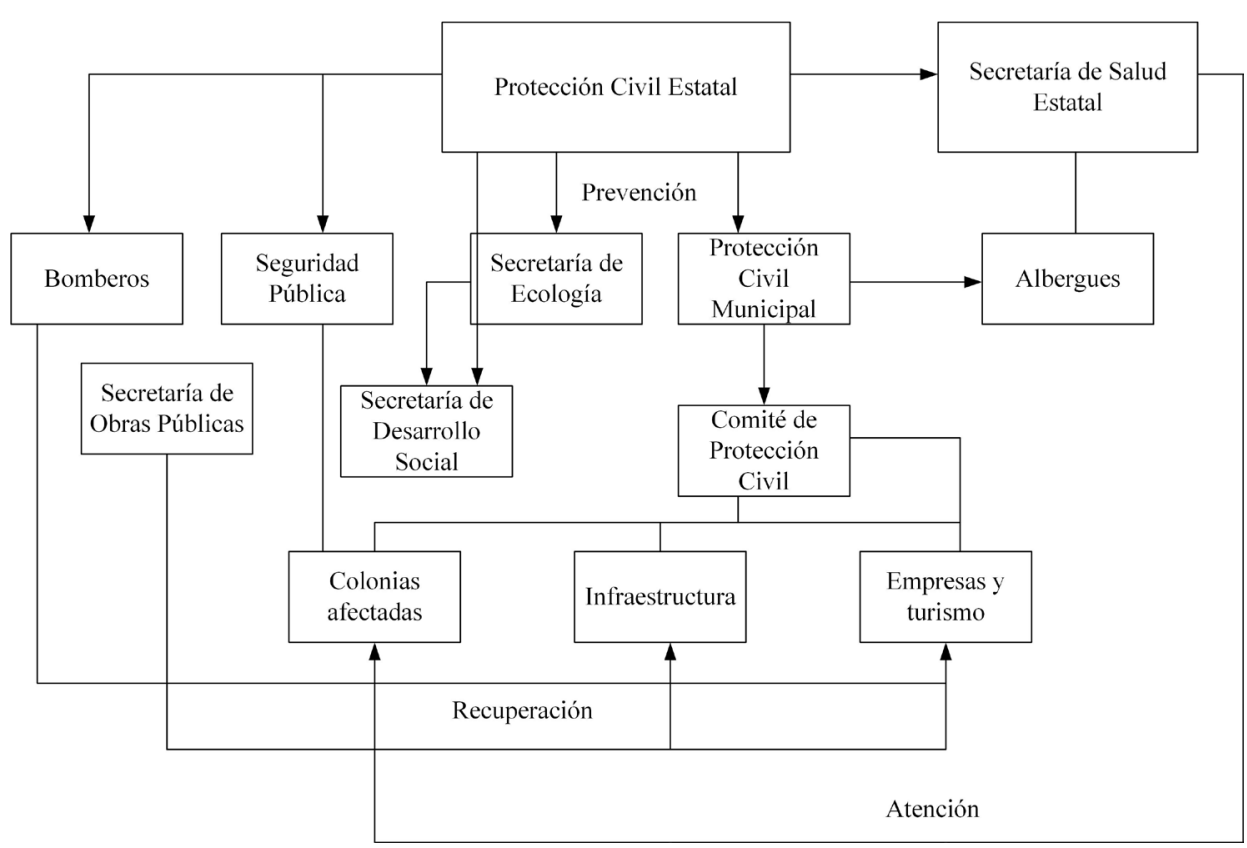

Figura 2. Gestión del riesgo del desastre y sus actores clave Fuente: Autora, 2018

La mayor limitación es la tecnología, existe la dificultad de obtener información de los municipios, de instituciones académicas que pueden apoyar a monitorear, expertos civiles que sean voluntarios y favorezcan la generación de bases de datos móviles. Protección Civil Estatal atiende paulatinamente y planifica de acuerdo a las prioridades, por su escaso personal, la comunidad les hace sentir que el apoyo es mínimo.

"Las instituciones no se han preocupado por tener información de todos los hechos que pasan, o sea, pasó un huracán, sí listo terminamos y se acabó, pero no hubo registro, no hubo evidencia fotográfica, no hubo la información de la expresión de la gente, ¿cómo lo vivió? ¿cómo lo pasó? Ninguna institución tiene ese antecedente. [...] Esas experiencias de vida que tuvieron, pero reales, preguntarles: ¿en dónde oíste que venía el huracán? ¿quién te informó? ¿a qué hora saliste de tu casa para irte al refugio? ¿con quién te refugiaste? Esas encuestas posteriores y, después agarrar y hacer unos folletos de información con las instituciones que están dedicadas, académicas y que hacen eso, para ir teniendo antecedente, para ir teniendo qué nos sirva. Y que se queden elementos, ahorita nosotros llegamos acá y lo primero que nos dicen: jno tenemos nada! Sí o no, a ver checa en el centro ¿qué dejaron?, entonces empezamos otra vez a generar información. [...] La administración anterior se llevó todo".

(Protección Civil Estatal, Entrevista, 2017)

Fue notable el reconocimiento de las autoridades locales y estatales sobre el uso mínimo de presupuesto federal que tiene el objetivo de la prevención, se enfatiza en el número de funcionarios para Protección Civil Estatal, sin embargo, también hace otros reconocimientos en cuanto a su 
función y atribuciones. Cabe mencionar, que el uso del presupuesto es menor, debido a que quienes aprueban los proyectos han priorizado acciones en otros estados.

"Mínimo personal que solo permite atención en el momento del desastre, casi no hay ciudadanos, no encontramos cómo llamar su atención, se hacen trípticos que concluyen siendo basura".

(Protección Civil Estatal, Entrevista, 2017)

Mencionan la necesidad de coordinación con otras instituciones y asociaciones sociales, a nivel local y estatal. Se reconoce que en mayo se reúne el comité para la protección civil, con una escasa participación social por la deficiente comunicación hacia la población, hay una existencia mínima de comités vecinales, la principal preocupación es la comunicación ineficiente, que la población no tiene interés, están dispuestos a recibir apoyo civil y económico, pero no hay presupuesto.

La falta de enlace para socializar la información y, una pérdida de recursos económicos por capacitación de personal, ya que la persona que asistió a los cursos, puede cambiar de trabajo y llevarse la información, lo cual es solo una inversión a fondo perdido. El efecto más pernicioso es que, se desconoce cómo acceder a presupuestos del Fondo para la Prevención de Desastres Naturales (FOPREDEN), para generar información, adquirir tecnología, ampliar el número de personas y sus equipos para atender emergencias, siendo un fondo presupuestal que México tiene contemplado en la agenda nacional, como un monto económico para la prevención de riesgos.

"Se hace uso, cuando existe un desastre, de cámaras de la Secretaría de Seguridad Pública del Estado de Quintana Roo (SSP), para observar las áreas inundadas y hacer un plan de atención".

(Protección Civil Municipal, Entrevista, 2017)

Existen ideas y propuestas sobre instrumentos tecnológicos en la mesa, sin que aún tengan respuesta de financiamiento. En Protección Civil Estatal, se explora la oportunidad de implementar una aplicación (app) para que las personas hagan uso de ella, que sirva como un relato del desastre al momento, así la población estará enterada de las áreas de peligro. Ya existen experiencias exitosas (Shibuya, 2017), en otras partes del mundo, lo que da pauta para asegurar que dicha estrategia marcaría un avance en la adaptación, como parte de la resiliencia ante huracanes.

Autores como Shibuya (2017) han dado seguimiento al uso de aplicaciones en internet que comunican situaciones de estrés en el momento oportuno. Es cier to que al instante del desastre el wifi es un servicio limitado, sin embargo, la creatividad puede ser utilizada para que, ideas de participación social a través de la tecnología actual construyan bases de datos sobre acontecimientos y formas de resolución.

En 2013 se hizo uso del presupuesto para elaborar el atlas de riesgo del estado en su Fase I. Peligro, Vulnerabilidad y Riesgo (Protección Civil, 2013). La Fase II está pendiente. Esto significa que existe un documento de identificación de riesgos rezagado y que, al elaborar una segunda fase, será obsoleto. Esto hace suponer que también existen algunos criterios de selección de proyectos y presupuesto desde la instancia de gobierno a nivel federal, limitando los esfuerzos de los gobiernos locales.

Protección Civil Estatal advierte la limitación de hacer partícipe a la población de informarse y crear comités vecinales que apoyen en los momentos de mayor estrés. Los folletos y carteles elaborados han sido minimizados por los funcionarios, distinguen el trabajo de la televisión y el radio, como medios básicos

Distinguen que se requiere actualizar la ubicación de albergues por hallarse en áreas de inundación, el crecimiento de la ciudad accionó nuevas áreas de inundación y, admiten que algunas escuelas que son albergues requieren de atención prioritaria, falta de servicios básicos, vidrios y ventanas dañados, sanitarios en condiciones lamentables e iluminación en las áreas comunes. Si el imaginario colectivo permitiera visualizar a las escuelas, como posibles albergues a futuro, tal vez el proceso de adaptación se construiría por un conjunto de acciones sociales de apropiarse de estos planteles $\mathrm{y}$, darles mantenimiento, cuidarlos, como a sus viviendas. 
Las necesidades básicas en los albergues solo permiten que las personas estén fuera de peligro de inundación. Sin embargo, se indicó que es mínima la privacidad, sin separación por sexos, sin sanitarios o rebasados por la cantidad de personas en el albergue. Protección Civil Estatal y la Secretaría de Salud Estatal, reconocen que existen protocolos de atención para las personas en los momentos de desastres. Incluso reconocidos a nivel nacional por la forma en que se comunican entre instituciones, movilizan enfermos o personas con necesidades médicas especiales, lo cual reduce el número de muertes y la vulnerabilidad física de las personas.

En el ámbito académico fue latente el desarrollo de trabajos que construyeron un proceso de adaptación y resiliencia, que se quedan en los estantes sin uso y sin difusión. Existen tesis de licenciatura, maestría y doctorado con mínima difusión, lo cual podría apoyar a la generación de información, abrir nuevos panoramas a los funcionarios sobre otros ejercicios institucionales de otros países, el conocimiento de la percepción de las personas cuando enfrentan un proceso de reconstrucción tras el desastre, entre otros.

En cuanto a los protocolos para la recuperación, solo hay dos importantes, el primero con la Secretaría de Salud Estatal para atender heridos, enfermos y epidemias. El segundo, con la Secretaría de Ecología del municipio de Othón P. Blanco, para limpiar calles, despejar los cables y postes, mover los espectaculares dañados y responder a los llamados de los vecinos sobre algún detalle con el arbolado. Los servicios básicos se van restaurando paulatinamente, sin coordinación con otra institución. Algunos entrevistados mencionaron que, a veces, se requiere el apoyo del ayuntamiento debido que algunas colonias o barrios permanecen sin servicio por diversos días.

La gobernanza climática global expresada por Demares (2016) implica que pueden realizarse posturas institucionales enfocadas a la comunicación hacia las personas y otras instancias gubernamentales, a fin de construir un proceso de adaptación, a través de la conciencia extensiva, introduciendo a la sociedad, en general, información sobre elevadas posibilidades de riesgo y la territorialización de los mismos. La forma en que los imaginarios colectivos de la prevención permean en la sociedad se irán introduciendo en la vida cotidiana de la población, modificando acciones tendientes a disminuir el riesgo. Territorializar las amenazas puede contribuir a la construcción social de nombrar y estigmatizar lugares por el desafío latente, que favorezca la adaptación y evitar acciones en lugares reconocidos como amenazantes, inestables, contingentes, violentos, etc. Tal como lo mencionan González y Torruco (2015), la identificación de las afectaciones al entorno ambiental construye áreas peligrosas, las personas pueden hacer uso de inferencias causales, por la expansión de la mancha urbana.

Es palpable que el proceso de adaptación derivada de los desastres es más forzoso que paulatino y planificado, haciendo uso de la experiencia social e institucional, con un avance significativo en cuanto a la forma en que las personas atienden por sí solas la protección de sus familias y viviendas. Algunos autores, como Sussan Cutter (2016) reconocen que, a pesar de que la academia produce diversa bibliografía sobre el tema, es mínimo el avance en la adaptación hacia la construcción de resiliencia. Incluso menciona que sobre vulnerabilidad social se ha disminuido el análisis, sin haber sido erradicada, hoy se escribe sobre resiliencia.

En cuanto a la infraestructura, algunos autores, como Fekete y sus colegas (2012 y 2015) han desarrollado algunos textos sobre las consideraciones sobre las líneas de abastecimiento de servicios básicos. En Chetumal, solo la CFE (Comisión Federal de Electricidad) ha hecho trabajos de actualización de las líneas de abastecimiento a nivel regional y local. Sin embargo, empresas como CAPA (Comisión de Agua Potable y Alcantarillado de Quintana Roo), empresas de televisión por cable, están ausentes en la coordinación con Protección Civil Estatal o Municipal para revisar continuamente sus redes. En estos procesos se demuestra que existe una falta de prevención en cuanto a la infraestructura, reduciendo las posibilidades de hablar de infraestructura crítica para la resiliencia. Esto significa que existen líneas de abastecimiento que requieren de una revisión y mantenimiento constante, para que al existir un riesgo la población solo se quede escasos días sin el servicio, ya que la recuperación se agiliza.

Los funcionarios expresaron que han sido las generaciones más envejecidas, aquellas que transmiten la información a sus descendientes, qué hacer, cómo hacerlo, en qué momento. Para Protección Civil Municipal, se torna imprescindible la oportunidad de generar bases de datos con esta información, así se podrían establecer estrategias reales, que permitan a las personas contar con un apoyo institucional acercado a sus necesidades, tal como lo expone Demares (2016). 
Los entrevistados evidenciaron la falta de conexión entre los documentos de planificación territorial y el atlas de riesgo, reconocen que a veces los documentos solo están para cumplir con un requisito, sin cumplir con su función normativa. En Protección Civil Estatal se acepta que los municipios tienen escasa participación al ser convocados para proveer información que aporte en la elaboración de atlas de riesgo. Así que la actualización de documentos para tomar decisiones y establecer políticas públicas, es más lento de lo que la ley establece. A pesar de que los acuerdos internacionales marquen las pautas para elaborar políticas en México sobre las ciudades y su gestión del riesgo de desastre, es menor el apoyo que se recibe del gobierno federal para hacer efectivo el proceso de adaptación que incluya acciones institucionales.

\section{CONCLUSIONES}

Los comentarios realizados por los funcionarios públicos abren oportunidades para elaborar documentos actualizados con información que debe ser privilegiada a fin de tomar decisiones y elaborar políticas y protocolos de actuación. Las ofertas del gobierno federal de contar con dos presupuestos para la prevención y atención a los desastres, deben ser utilizadas por los gobiernos locales a fin de favorecer la gestión del riesgo de desastre causados por los huracanes, se requiere de un esfuerzo social para concientizar sobre el beneficio y el ahorro futuro de utilizar correctamente estos presupuestos.

Las ciudades costeras de Quintana Roo, presentan un panorama de grupos vulnerables que va en aumento, la migración nacional y la llegada de inversionistas y vacacionistas, nacionales e internacionales, genera la presión por contar con instrumentos políticos de actuación a fin de hacer a las ciudades costeras resilientes. También de hacer énfasis en el engrosamiento de instituciones locales y estatales que son protagonistas de la gestión del riesgo de desastre, así como de herramientas que informen a los visitantes sobre los protocolos de actuación en caso de acercarse un huracán.

Se tiene el imaginario colectivo de ser una ciudad con cultura arraigada para enfrentar a los huracanes, no obstante, las autoridades reconocen que aún las instituciones y la sociedad están lejanas de contar con planes más allá de la atención y la recuperación. Hace falta construir acciones de prevención, mitigación y reconstrucción. Tanto Busso (2012), como Nilakant y sus colegas (2014) justifican la necesidad de contar con una comunicación efectiva entre instituciones y población. Los intereses de partidos políticos han influido en disminuir la capacidad de respuesta en la gestión del riesgo de desastre, por la falta de cooperación y corresponsabilidad, lo que permite suponer que los presupuestos y trabajos realizados se van a un fondo perdido.

Es necesario capacitar a un número mayor de personal, elaborar instrumentos vigentes derivados de las experiencias que permitan construir redes competentes de colaboración para ser el garante de accionar protocolos de apoyo, otorgando algunas responsabilidades jurídicas transversales para la construcción de resiliencia en las ciudades del caribe mexicano. Al parecer las áreas de mayor riesgo y absorción de presupuestos son los asentamientos, legales e ilegales, en áreas de susceptibilidad ambiental.

Las limitaciones más apremiantes son la comunicación efectiva con la población y el uso de la tecnología para generar bases de datos históricas y SIG para consulta general, esto representa un desconcierto sobre la utilización de los presupuestos para la prevención de desastres.

Al hablar de la infraestructura crítica es indispensable estar de acuerdo con algunos autores (Cutter et al., 2010 y Fekete et al., 2012), a fin de permitir que las personas pasen por la fase de recuperación y reconstrucción sin la pérdida total o parcial de abastecimiento de agua y energía eléctrica, sobre todo, mantener un estado de comunicación y de seguridad para los habitantes. Hasta ahora, la falta de revisión, mantenimiento y actualización de la infraestructura, conducirá a inundarse en heces, multiplicando los riesgos a la salud.

Los resultados expuestos derivan de un estudio exploratorio sobre la gestión del riesgo de desastre en ciudades costeras, ya que, a pesar de existir reuniones mundiales, la forma en que una comunidad se verá afectada, el presupuesto, las políticas públicas y la cultura, son determinantes en la forma de enfrentar, recuperarse y adaptarse a la continuación de su vida en su ciudad. Lo fundamental es eliminar la postura de las comunidades y sus instituciones ante los planes de desarrollo urbano, ordenamiento territorial y atlas de riesgo, convendría utilizarlos como políticas 
públicas que contribuyan a la reducción del riesgo de desastre por la información que contienen. Las ciudades costeras se vislumbran como los escenarios futuros de las grandes aglomeraciones, por lo que, detenerse en este momento y, re-direccionar la expansión urbana, podría salvar vidas, lograr un desarrollo urbano sostenible $y$, una mayor resiliencia mundial.

Algunos autores (Nilakant et al., 2014, Chan et al., 2014) mencionan que son, la recuperación y reconstrucción los factores determinantes en el proceso de adaptación, en países en desarrollo la dirección de la gestión del riesgo apunta a resolver los problemas estructurales que construyen amenazas, sin embargo, en México, los ajustes se han realizado en la atención, es el momento en que todos están expuestos, las respuestas institucionales han sido entregas tardías de despensas, colchonetas y ubicación de albergues. La recuperación es solo dar continuidad al funcionamiento de servicios suspendidos por seguridad, agua potable, energía eléctrica, comunicaciones, son las personas quienes establecen sus propias redes de apoyo y negociación para encontrar sustento jurídico para la obtención de créditos o préstamos económicos a fin de resolver la reconstrucción de sus viviendas.

Hasta ahora, a pesar de la legislación, parece que la resiliencia es un proceso paulatino y con limitaciones, la permisividad es uno de los factores que favorecen acciones peligrosas, sin responsables jurídicos, se desarrolla un proceso de adaptación de la población a perder fauna, flora, ecosistemas y al aumento de su fragilidad al entorno. Cambios extremos de temperatura en invierno y verano, lluvias atípicas, nuevas áreas inundables. Se deja la prevención y la mitigación con escasos procesos de adaptación, se enfoca la gestión del riesgo de desastre en atender y recuperar los servicios básicos, aún se está lejos de apoyar a la sociedad a la reconstrucción con un proceso bien estructurado a fin de mitigar futuros riesgos y construir resiliencia.

Los funcionarios entrevistados mencionaron una limitación importante en la gestión del riesgo, la mínima preocupación de la sociedad por asegurar los bienes materiales, participar e informarse, las convocatorias para voluntarios civiles que apoyen en la recuperación siempre han sido eventos formales sin respuesta. Hasta el momento del desastre, la población por sí misma, sale a la calle para solidarizarse a la limpieza de calles, sin abandonar sus hogares frente a la inseguridad social y, observando los manejos indiscretos del reparto de víveres y bienes materiales, como un signo de recuperación.

Existen elementos sustanciales sobre la gestión del riesgo de desastre, a pesar de que se reconocen avances paulatinos, está presente el uso de información por experiencias anteriores, la construcción de protocolos de actuación que son admirados por los turistas en el caribe mexicano, la evacuación y la atención en los albergues. Tanto la resiliencia, como la adaptación son procesos progresivos, la integración de una gestión del riesgo enfocada a la integración de la sociedad y de los mismos empresarios puede reforzar la resiliencia, construir un proceso de adaptación continuo donde las personas sepan qué hacer y cómo responder, con amplias opciones de información, noticias, confianza en las instituciones, liderazgos, todo ello hacia una reestructuración cultural ante los desastres.

\section{AGRADECIMIENTOS}

Este trabajo es parte de un proyecto financiado por CONACYT 248375: Resiliencia en ciudades costeras Del Caribe Mexicano ante desastres por huracanes: Chetumal, Tulum y Playa del Carmen.

\section{REFERENCIAS}

Balcu, C.L. (2010). Environmental Influence on Natural and Anthropogenic Disasters, Revista Academiei Fortelor Terestre Nr., 3 (59), 383-387.

Brouwer, R., Akter, S., Brander L. \& Haque E. (2007). Socioeconomic Vulnerability and Adaptation to Environmental Risk: A Case Study of Climate Change and Flooding in Bangladesh, Risk Analysis, 27 (2), 313-326.

Busso, G. (2012). Vulnerabilidad y Exclusión Urbana. Las Variables de Población en las Políticas de Desarrollo Local en Ciudades de Tamaño Intermedio. En Egea, C. Sánchez, D. \& Soledad, J. (Eds.), Vulnerabilidad Social. Posicionamientos y Ángulos desde Geografías Diferentes. Granada: Colecciones Eirene, Universidad de Granada (pp.91-112).

Chan, S.L., Wey, W.M. \& Chang, P.H. (2014). Establishing Disaster Resilience Indicators for Tan-sui River Basin in Taiwan, Social Indicators Research, 115 (1), 387-418. 
Chan, N.W., Zakaria, N.A., Ab Ghani, A. and Lian T.Y. (2004). Integrating Official and Traditional Flood Hazard Management in Malasya. En 1st. International Conference on Managing Rivers in the 21 st. Century. Issues \& Challenges. Penang, Malaysia: USM (pp. 409-419).

Cutter, S.L., Boruff, B.J. \& Shirley, W.L. (2003). Social Vulnerability to Environmental Hazards, Social Science Quarterly, 84 (2), 242-261.

Cutter, S.L., Burton, C.G. \& Emrich, C.T. (2010). Disaster Resilience Indicators for Benchmarking Baseline Conditions, Journal of Homeland Security and Emergency Management, 7(1), Article 51. DOI: 10.2202/1547-7355.1732

Cutter, S.L. (2016). Our Hazardous Environment: Four Decades of Progress or Retrenchment? Environment, Science and Policy for Sustainable Development, 58 (1), 2-3.

Demares, M. (2016). La importancia de la gobernanza climática global y de la vigésimo primera Conferencia de las Partes en la lucha contra el Cambio Climático, Observatorio Ambiental, 19, 55-69. DOI: http://dx.doi.org/10.5209/OBMD.54158

Elliott, J.R., Haney, T.J. \& Sams-Abiodun, P. (2010). Limits to Social Capital: Comparing Network Assistance in two New Orleans neighborhoods devastated by Hurricane Katrina, The Sociological Quarterly, 51, 624-648.

Fekete, A., Lauwe, P. \& Geier, W. (2012). Risk management goals and identification of critical infrastructures, Journal Critical Infraestructures, 8 (4), 336-353. DOI: 10.1504/IJCIS.2012.050108

Fekete, A., Tzavella, K., Armas, I., Binner, J., Garschagen, M., Giupponi, C., Mojtahed, V., Pettita, M., Schneiderbauer, S. \& Serre, D. (2015). Critical Data Source; Tool or Even Infrastructure? Challenges of Geographic Information Systems and Remote Sensing for Disaster Risk Governance, ISPRS Int. J. Geo-Inf., 4, 1848-1869.

Freudenberg, M. (2003). Composite Indicators of Country Performance: A Critical Assessment, OECD Science, Technology and Industry Working Papers, 2003/16. Paris: OECD Publishing. DOI: http:// dx.doi.org/10.1787/405566708255

González, M.A. \& Torruco, D. (2015). Perfiles sociales y económicos en la costa de Quintana Roo: su relación con el turismo de playa, Investigación ambiental, 7 (1), 1-11.

Gupta, A., Tyagi, P. \& Sehgal, V. (2011). Drought disaster challenges and mitigation in India: strategic appraisal, Current Science, 100 (12), 1795-1806.

Ihl, T. \& Frausto, O. (2014). El cambio climático y los huracanes en la Península de Yucatán. En Frausto, O. (Ed.), Monitoreo de riesgo y desastre asociados a fenómenos hidrometeorológicos y cambio climático. Chumatal, México: Universidad de Quintana Roo (pp. 42-49).

Instituto Nacional de Ecología INE. (200o). La calidad del agua en los ecosistemas costeros de México. Ciudad de México: INE \& Semarnap. Disponible en: http://e-mar.sct.gob.mx/fileadmin/biblioteca/ ecologia/308.pdf [Visitada el 3 de mayo del 2014].

Instituto Nacional de Estadística y Geografía INEGI. (2016). Marco Geoestadístico. Ciudad de México: INEGI. Disponible en: http://www.beta.inegi.org.mx/app/mapas/ [visitada el 5 de marzo de 2018]

Panel Intergubernamental sobre Cambio Climático IPCC. (2007). Climate Change 2007: Climate Change Impacts, Adaptation and Vulnerability. En Parry, M., Canziani, O., Palutikof, J., van der Linden, P. \& Hanson, C. (Eds.), Cuarto Informe de Evaluación del Grupo Intergubernamental de Expertos sobre el Cambio Climático (IPCC). Cambridge: Cambridge University Press.

Jonkman, S.N., Maaskant, B., Boyd, E. \& Levitan, M.L. (2009). Loss of Life Caused by the Flooding of New Orleans After Hurricane Katrina: Analysis of the Relationship Between Flood Characteristics and Mortality, Risk Analysis, 29 (5), 676-698.

Kelly, P.M. \& Adger W.N. (200o). Theory and Practice in Assessing Vulnerability to Climate Change and Facilitating Adaptation, Climatic Change, 47, 325-352.

Leyva, J.F. (2016). Régimen de Responsabilidad y Mecanismos Jurídicos para la reparación del daño ambiental, Observatorio Medioambiental, 19, 111-131. DOI: http://dx.doi.org/10.5209/OBMD.54163

Morrison, T.H., Adger, W.N., Brown, K., Lemos, M.C., Huitema, D. \& Hughes, T.P. (2017). Mitigation and adaptation in polycentric systems: sources of power in the pursuit of collective goals, WIREs Climate Change, e479, 1-16. DOI: 10.1002/wcc.479

Nilakant, V., Walker, B., van Heugen, K., Baird, R. \& de Vries, H. (2014). Research Note: Conceptualising Adaptive Resilience using Grounded Theory, New Zealand Journal of Employment Relations, 39 (1), $79-86$. 
Organización de las Naciones Unidas ONU. (2016). Nueva Agenda Urbana. Quito \& Nueva York: ONU.

Østergaard Nielsen, J. \& Reenberg, J. (2010). Cultural barriers to climate change adaptation: A case study from Northern Burkina Faso, Global Environmental Change, 20, 142-152.

Programa de las Naciones Unidas para el Desarrollo PNUD. (2014). Gestión del Riesgo de Desastres ¿Qué hace el PNUD en Gestión del Riesgo de Desastres en América Latina y el Caribe? Panamá: CPRPNUD América Latina y el Caribe.

Protección Civil de México. (2013). Recursos Autorizados. Ciudad de México: Gobierno de México: Disponible en: http://www.proteccioncivil.gob.mx/work/models/ProteccionCivil/Resource/2254/2/ images/PROYECTOS\%2oFOPREDEN\%20AUTORIZADOS\%202013.pdf [visita el 9 de febrero de 2018].

Riffo, L.P. (2013). Situación y perspectivas del desarrollo regional en América Latina y el Caribe. En XXXI Reunión plenaria. Foro permanente de direcciones de presupuesto y finanzas de la República de Argentina. Buenos Aires: ILPES-CONEVAL (pp. 1-65).

Shibuya, Y. (2017). Mining Social Media for Disaster Management: Leveraging Social Media Data for Community Recovery. En 2017 IEEE International Conference on Big Data (BIGDATA). Boston, MA.: IEEE. DOI: 10.1109/BigData.2017.8258286

Vergara, W., Ríos, A.R., Galindo, L.M., Gutman, P., Isbell, P., Suding, P.H. \& Samaniego, J.L. (2014). El Desafío Climático y de Desarrollo en América Latina y el Caribe Opciones para un desarrollo resiliente al clima y bajo en carbono. Washington D.C.: CEPAL, BID \& WWF. 